\title{
Letter to the Editor concerning "Indirect foraminal decompression and improvement in the lumbar alignment after percutaneous cement discoplasty" by Laszlo Kiss et al. (Eur Spine J; 28(6):1441-1447)
}

\author{
Haifeng Wang ${ }^{1} \cdot$ Zuowei Wang $^{2}$
}

Received: 19 July 2019 / Revised: 19 July 2019 / Accepted: 13 September 2019 / Published online: 28 September 2019

○) Springer-Verlag GmbH Germany, part of Springer Nature 2019

We have read with great interest the recent article on indirect foraminal decompression and improvement in the lumbar alignment after percutaneous cement discoplasty (PCD) by Kiss et al. The article reveals that PCD can provide a foraminal decompression and lumbar alignment correction effect. We appreciate the authors' work on this topic. Although it was a well-performed technique note, we still seek clarification on few points regarding the message being sent out by the article and to present our viewpoint.

First, in Table 2 (results of radiological measurements), the mean change of L1-5 lumbar lordosis (LL) of pre- and post-operation was $3.4^{\circ}$ without statistically significant difference $(P+0.5)$, but the mean changes of segmental lordosis, segmental lordosis with PCD, and segmental lordosis without PCD were $2.2^{\circ}, 1.5^{\circ}$, and $3.1^{\circ}$, respectively, and all these with statistical significance $(P<0.5)$. As L1 -5 lumbar lordosis was caused by the lordosis of each segment cumulatively, how to interpret the inconsistency between the L1-5 lumbar lordosis and segmental lordosis?

Second, in statistical analysis, in case of the spinopelvic radiological parameters, one-way repeated measures ANOVA and nonparametric Friedman test were conducted to determine the statistically significant differences over the course of a 6-month follow-up period. A $P$ value less than 0.05 was considered significant. Table 2 demonstrates that pelvic incidence (PI), sacral slope (SS), pelvic tilt (PT), and L1-5 lumbar lordosis (LL) were normal distributed data and other spinopelvic radiological

Zuowei Wang

wzw6855@163.com

Haifeng Wang

haifengwang006@163.com

1 Department of Neurosurgery, Beijing Hospital, National Center of Gerontology, Beijing, China

2 Department of Neurosurgery, Xuanwu Hospital, Capital Medical University, No.45 Changchun Street, Xicheng District, Beijing 100053, China parameters besides these were non-normal distribution. It seems that difference of normal data was tested by one-way repeated measures ANOVA, and non-normally distributed data were tested by nonparametric Friedman test. One-way repeated measures ANOVA and nonparametric Friedman test were suitable to test the difference between three or more groups, and repeated ANOVA needs to meet the assumption of sphericity. However, to be exact, the one-way repeated measures ANOVA and nonparametric Friedman test were applied to test the difference between post-operation and pre-operation or between 6-month follow-up and post-operation; these were post hoc tests. When performing post hoc multiple tests, the $P$ value might be more appropriate to be adjusted by Holm-Bonferroni method to reduce the possibility of type I error. Whether the $P$ value in the article has all been adjusted? And in statistical description, central tendency of non-normally distributed data should be described as interquartile ranges and medians. We are concerned that the article may apply incorrect statistical methods to draw wrong conclusions.

We think the author should provide more information and modify the statistical method. Despite these concerns, the authors' paper has demonstrated that the minimally invasive PCD surgery can not only have a segmental stabilizing effect, but also provide a foraminal decompression and lumbar alignment correction effect. We are looking forward to further studies from the authors.

Author contributions HW wrote the manuscript. Prof. ZW critically revised the manuscript.

\section{Compliance with ethical standards}

Conflict of interest The authors declared no conflict of interest.

Publisher's Note Springer Nature remains neutral with regard to jurisdictional claims in published maps and institutional affiliations. 